\title{
The morphological and morphometric analysis of the variant patterns of the tricipital aponeurosis: a new anatomical classification with possible clinical implications
}

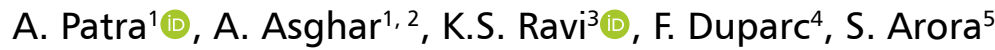 \\ ${ }^{1}$ All India Institute of Medical Sciences, Bathinda, Department of Anatomy, AlIMS, Bathinda, India \\ ${ }^{2}$ All India Institute of Medical Sciences, Patna, India \\ ${ }^{3}$ All India Institute of Medical Sciences, Rishikesh, India \\ ${ }^{4}$ Universite de Rouen, France \\ ${ }^{5}$ Maulana Azad Medical College, Department of Anatomy, Bahadur Shah Zafar Marg,, New Delhi, India \\ [Received: 24 August 2021; Accepted: 10 September 2021; Early publication date: 28 September 2021]
}

Background: Tricipital aponeurosis (TA) has gained attraction as a constant and reliable landmark to identify the location of radial nerve in the setting of fracture distal humeri. The aponeurosis itself shows variant anatomical patterns. In this study, we intend to provide a comprehensive description and functional classification of observed anatomical variations with possible clinical implications.

Materials and methods: Sixty arms belonging to 30 adult cadavers were studied. TA was examined grossly to document variations in its shape and classified accordingly. Subsequently, length and breadth of TA were measured. The distance of the radial nerve (RN) from the point of confluence and from the lateral border of TA was also measured (tricepso-radial distance [TRD]). These distances were correlated with the different patterns of TA obtained.

Results: Based on the shape of the proximal apex of TA or point of confluence and frequency of their occurrence, we propose a new classification of 4 patterns for the TA anatomy. Pattern I: classically seen as the triangular proximal apex (76.67\%); pattern II: tongue shaped or blunt proximal apex (18.33\%); pattern III: bifurcated or dual proximal apex (3.33\%); pattern IV: as the absence of TA (1.67\%). The mean of length and breadth of TA was $16.58 \pm 2.05 \mathrm{~cm}$ and $3.61 \pm 0.61 \mathrm{~cm}$, respectively. The mean distance of $R N$ from point of confluence and lateral border of TA was $3.57 \pm 0.19 \mathrm{~cm}$ and $2.04 \pm 0.56 \mathrm{~cm}$, respectively. The length, breadth of TA and TRD differs amongst the different patterns of TA.

Conclusions: Anatomical variations in the shape and size of TA are frequently encountered. The proposed, hitherto undescribed, classification may make operating surgeon aware of these morphological variations and help prevent iatrogenic injury to RN. Such classification is simple and unique; however, its success relies upon universal acceptance. (Folia Morphol 2022; 81, 4: 1022-1030)

Key words: triceps brachii, aponeurosis, classification, humerus, fracture, landmark 


\section{INTRODUCTION}

Fracture of the humeral shaft constitutes $1-3 \%$ of all the fractures [27]. Fractures involving the distal third of the humerus require surgical intervention to achieve skeletal stability [17, 20,26]. Several surgical approaches have been described, such as anterior, anterolateral, lateral, medial, and posterior triceps splitting approaches [16]. Amongst them, the posterior approach (triceps splitting) offers excellent exposure of humeral diaphysis and distal metaphysis [31]. However, such intervention may injure the radial nerve (RN). The long tortuous course and proximity with the periosteum make the RN very prone to injury. RN palsies are mainly two types, primary and secondary. Primary palsies are those mostly due to fracture humeral shaft and include $11.8 \%$ of all cases reported [26]. Secondary or iatrogenic palsies can occur during any kind of operative interventions for unstable humeral fractures, such as open reduction and internal fixation with plating or intramedullary nailing $[4,6,8,10,11]$. These iatrogenic palsies are not uncommon in routine practices and are of major concern for the protection of the radial nerve [29]. The radial nerve must be identified and mobilised from the operative field throughout the procedure [11]. Identification of the nerve is critical, some studies have established the anatomic relationship of the $\mathrm{RN}$ with various bony landmarks, such as medial epicondyle, lateral epicondyle of humerus and tip of the acromion $[4,6,8,10,11]$. Previously orthopaedic surgeons used to depend on these landmarks to identify the RN during surgical exploration of the humerus but the wide range of these proposed anatomic relationships may pose trouble in localising the RN during surgery. Moreover, such relationships with bony landmarks may not hold true in clinical settings, especially with fractures of the distal third of the humerus [6]. The 'proximal apex' or 'the point confluence' of tricipital aponeurosis appear to be more consistent and reliable anatomic landmarks to locate the RN more precisely during the 'triceps splitting' approach to the humerus $[8,27]$. On the other hand, the location of the nerve immediately adjoining the lateral border of aponeurosis $(<1.00 \mathrm{~cm})$ can be considered "safe" for soft tissue dissection while surgically approaching distal humeral fractures through lateral approach [20,24]. However, the anatomical variations in the shape and dimensions of the tricipital aponeurosis (TA) have not been described in detail despite these details are imperative for an orthopaedic surgeon to know [19].
Such variations are pivotal in its universal acceptance as a reliable surgical landmark. With such context, our primary aim was to document various shapes, sizes of $\mathrm{TA}$, and measure the distance of RN from pre-determined landmarks of TA (proximal apex or confluence of TA, lateral border of TA). The secondary aim was to propose a new classification for the TA anatomy based on variant patterns attained and correlate them with the measured variables.

\section{MATERIALS AND METHODS}

Sixty upper limbs belonging to 30 formalin embalmed cadavers ( 23 male, 7 female) aged between 45 to 60 years with a mean age of $55.84 \pm 7.74$ years were examined in this study. We have used $10 \%$ formalin solution for body preservation as it may be considered as a standardized method of fixation with minimal tissue hardening and volume shrinkage effect. Arms having any gross malformation/deformity or signs of the previous injury were excluded from the study. The specimens were prepared through scapula-thoracic disarticulation and midclavicular amputation. Each cadaveric upper extremity was placed at 90-degree flexion at the elbow joint as this is the most common position used intraoperatively. A posterior midline skin incision starting from the tip of the acromion up to the tip of the subcutaneous olecranon was given. Subcutaneous tissue and deep fascia were also incised in the same line exposing the triceps muscle. The shiny TA was immediately visible on the posterior arm inserted into the posterior rough area of the subcutaneous olecranon. The TA represents the convergence of the long and lateral heads of the triceps muscle bellies, forming a shiny layer lying between the deep fascia superficially and periosteum of the distal humeral dia-metaphyseal segment underneath. It can be readily identified by its shiny pearl white colour. Proximally, the TA runs obliquely upwards over the posterior aspect of the arm, creating two apices, one proximal (main), the other distal or lateral. The proximal apex is also known as the point of confluence (the meeting point of two heads of triceps with the proximal end of the TA). Wide variations were noted in the shape of the proximal apex of the TA. Based on the observations and their frequency of occurrence, we propose a new classification of 4 patterns for the TA anatomy. The pattern I: classical variant with the triangular proximal apex coincides with the point of confluence; pattern II: tongue-shaped or blunt proximal apex coincides with 


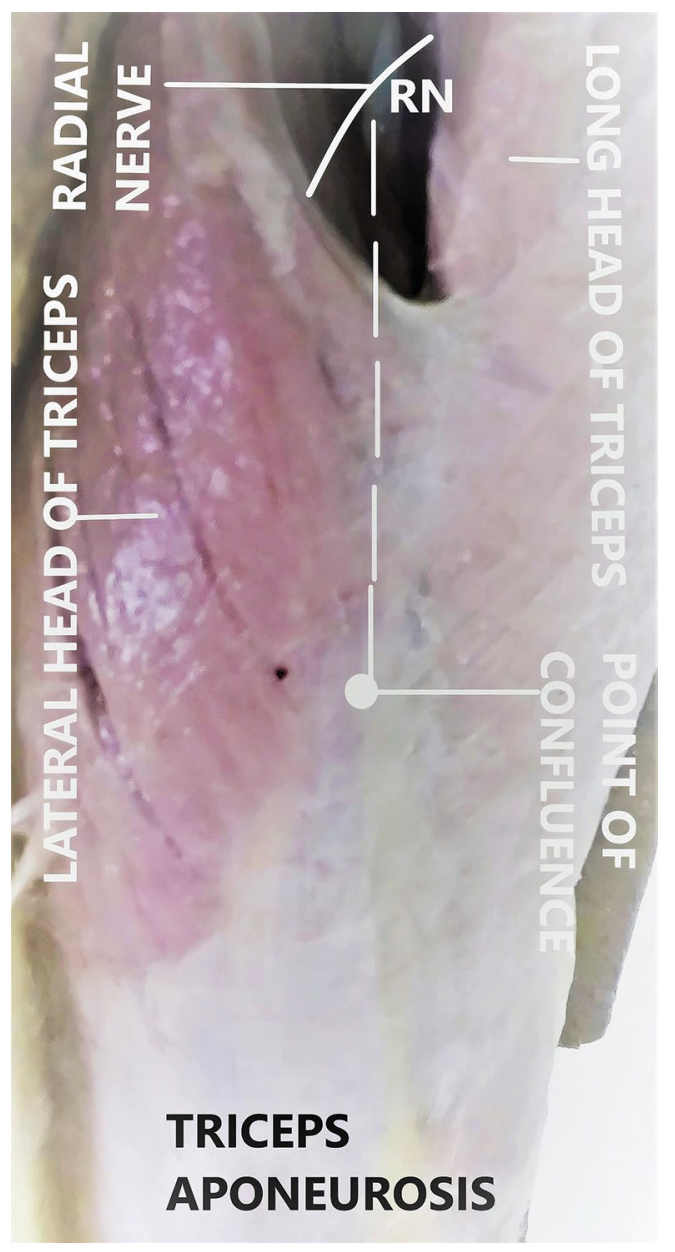

Figure 1. Measurement of tricepso-radial distance posterior approach; straight distance between point of confluence and radial nerve (RN) (dotted line).

the point of confluence; pattern III: bifurcated or double triangular proximal apex does not coincide with the point of confluence; pattern IV: as the absence of TA. After noting the various shapes, we have measured the length and breadth of TA by the methods described ahead. The straight distance between the point of confluence of TA (A) to the tip of the olecranon was measured and recorded as length. We have marked the proximal or medial apex as point ' $A$ ' (in the majority of cases 'point of confluence' coincides with proximal apex) and distal or lateral apex as point ' $B$ '. The breadth of TA was not uniform throughout its whole length. So, we have measured the breadth at 3 equidistant points splitting the length of TA into 4 equal segments. Proximally, the first point was at the level of the distal apex (B), followed by 2 more equidistant points along the length. The mean of these

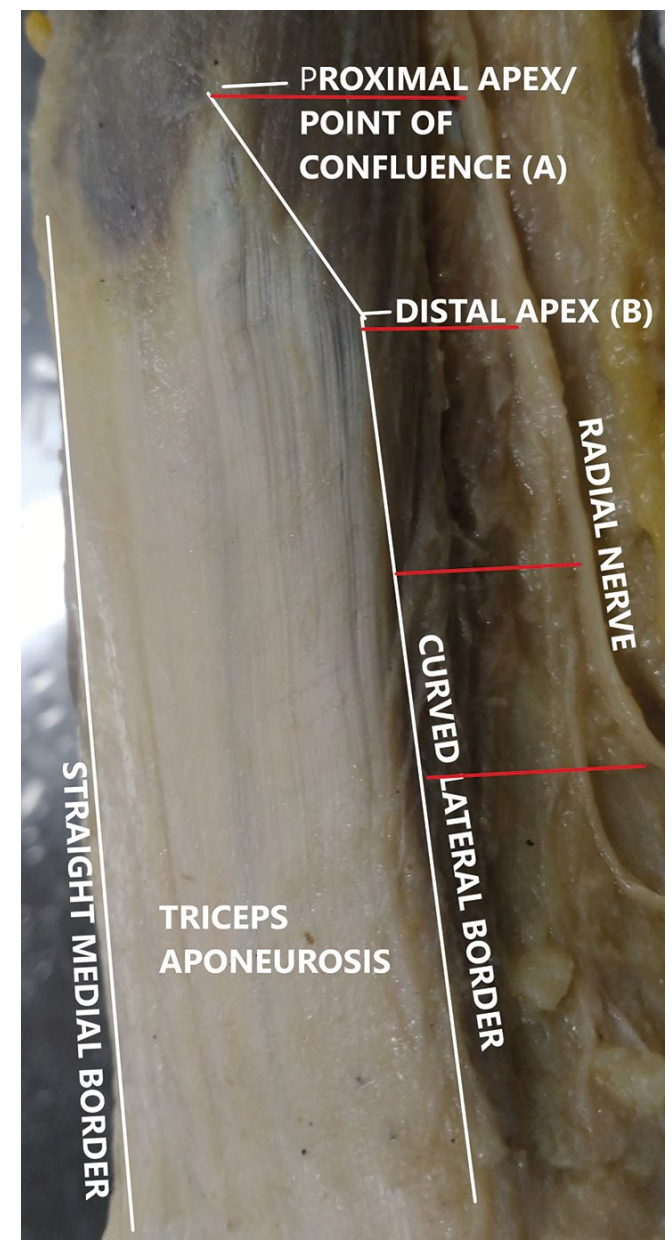

Figure 2. Measurement of tricepso-radial distance in lateral approach; distances between the lateral border of tricipital aponeurosis and radial nerve measured at four equidistant points (red marked line) along lateral its border and mean were taken.

3 distances was taken as the average breadth of TA. Subsequently, the distance of the RN from the point of confluence and lateral border of TA was also measured (tricepso-radial distance [TRD]) (Figs. 1, 2). All the measurements were taken by the same individual, using digital vernier callipers (Mitutoyo, Japan) with the least count of $0.01 \mathrm{~mm}$. The measurements were repeated 2 times, and the mean was taken as final for further analysis. Results were expressed as mean \pm standard deviation. Correlation analysis was done between the distances measured (length, breadth of TA and TRD) and various patterns of TA obtained.

\section{Statistical analysis}

Subsequent statistical analysis through paired, 2-tailed Student t-test was performed (Microsoft Excel 2007; Microsoft, Redmond, WA, USA). 


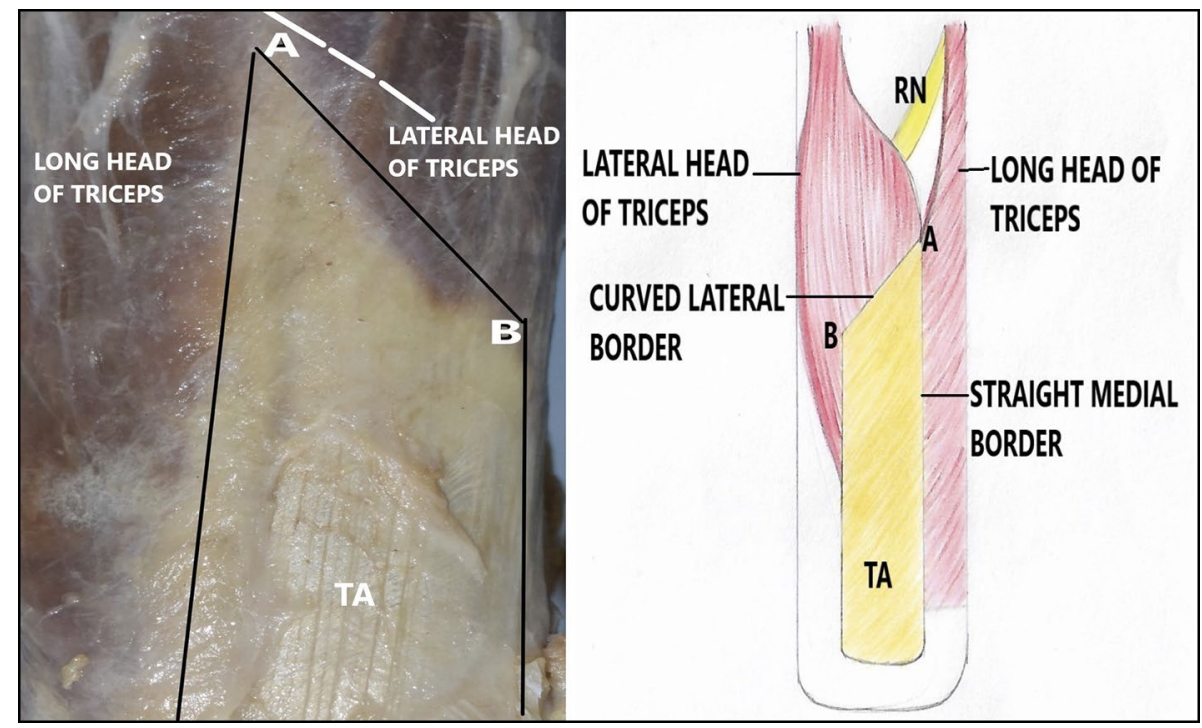

Figure 3. Classical variant of tricipital aponeurosis (TA) with the triangular proximal apex (A). Proximal apex coincides with confluence of TA. Medial border is straight while lateral border is oblique or curved. Dotted line showing the location of radial nerve (RN) after separation of the two heads of triceps. (Schematic drawing is the mirror images of the actual photograph); B — distal apex.

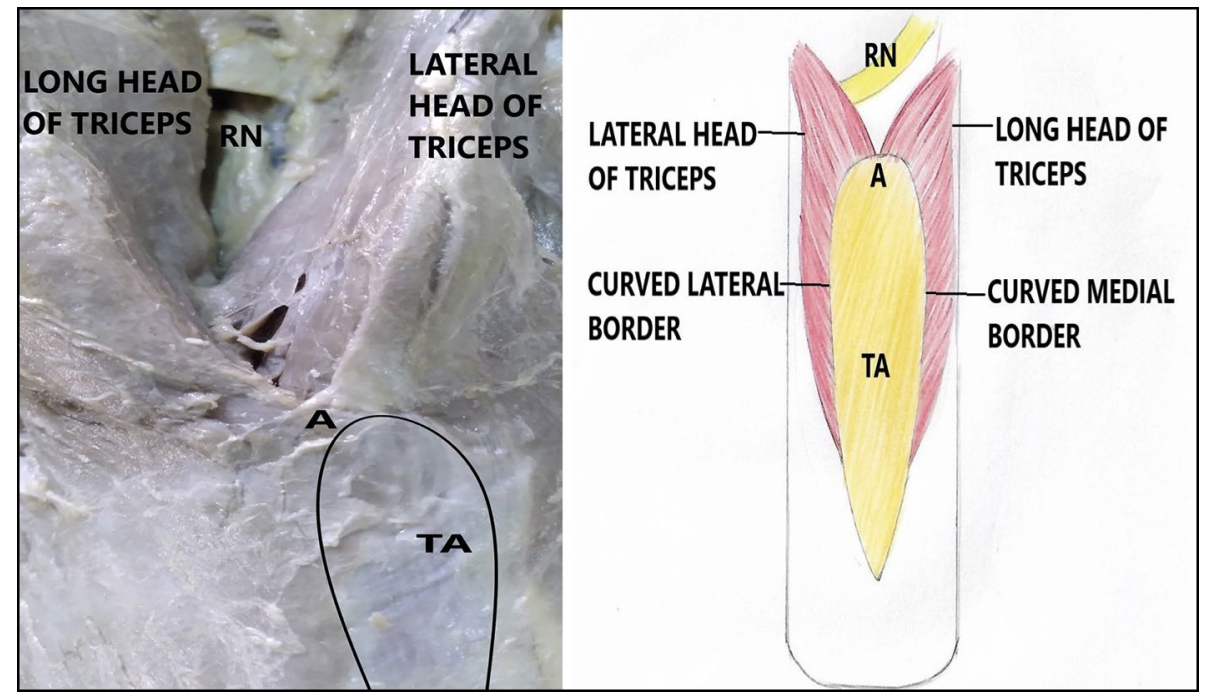

Figure 4. Tongue shaped or blunt proximal apex $(A)$ coincides with point of confluence. Both the medial and lateral border are oblique or curved. (Schematic drawing is the mirror image of the actual photograph); $\mathrm{RN}$ - radial nerve; $\mathrm{TA}$ - tricipital aponeurosis.

\section{RESULTS}

Based on the observations and the frequency of occurrence of anatomical variations in the shape and size of the proximal apex of TA, we observed 4 different patterns: pattern I: classical variant with the triangular proximal apex coincides with the point of confluence; pattern II: tongue-shaped or blunt proximal apex coincides with the point of confluence; pattern III: bifurcated or double triangular proximal apex does not coincide with the point of confluence; pattern IV: as the absence of TA.

The classical variant or pattern I was observed most commonly (76.67\%; Fig. 3), followed by pattern II (18.33\%; Fig. 4) and pattern III (3.33\%; Fig. 5). Pattern IV was found in only 1 specimen (1.67\%; Fig. 6). In this solitary specimen, the 2 heads of the triceps were inserted into the fibrous intramuscular septum which carried the insertion downward up to the tip 


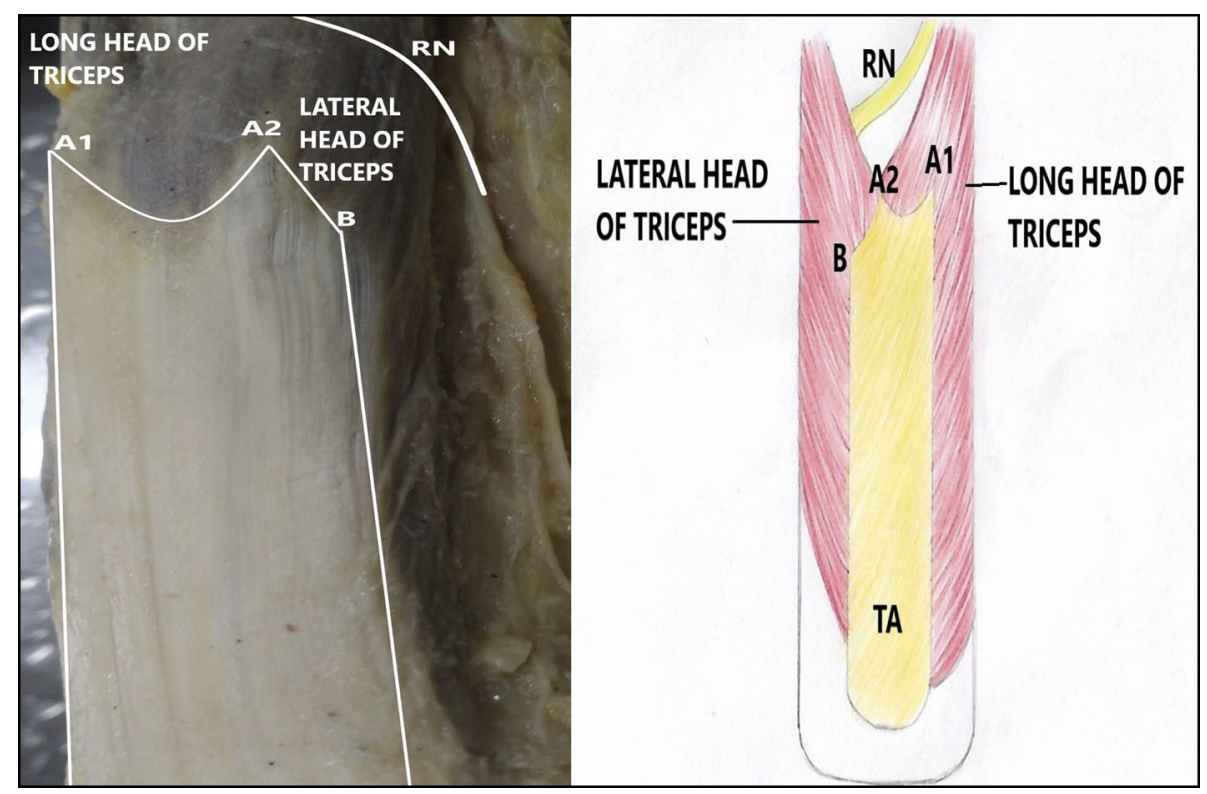

Figure 5. Bifurcated or double triangular proximal apex. A1 denotes the medial proximal apex; A2 denotes the lateral proximal apex coincides with confluence of aponeurosis. Medial border is straight while lateral border is oblique or curved. (Schematic drawing is the mirror image of the actual photograph); B — distal apex; RN — radial nerve; TA — tricipital aponeurosis.

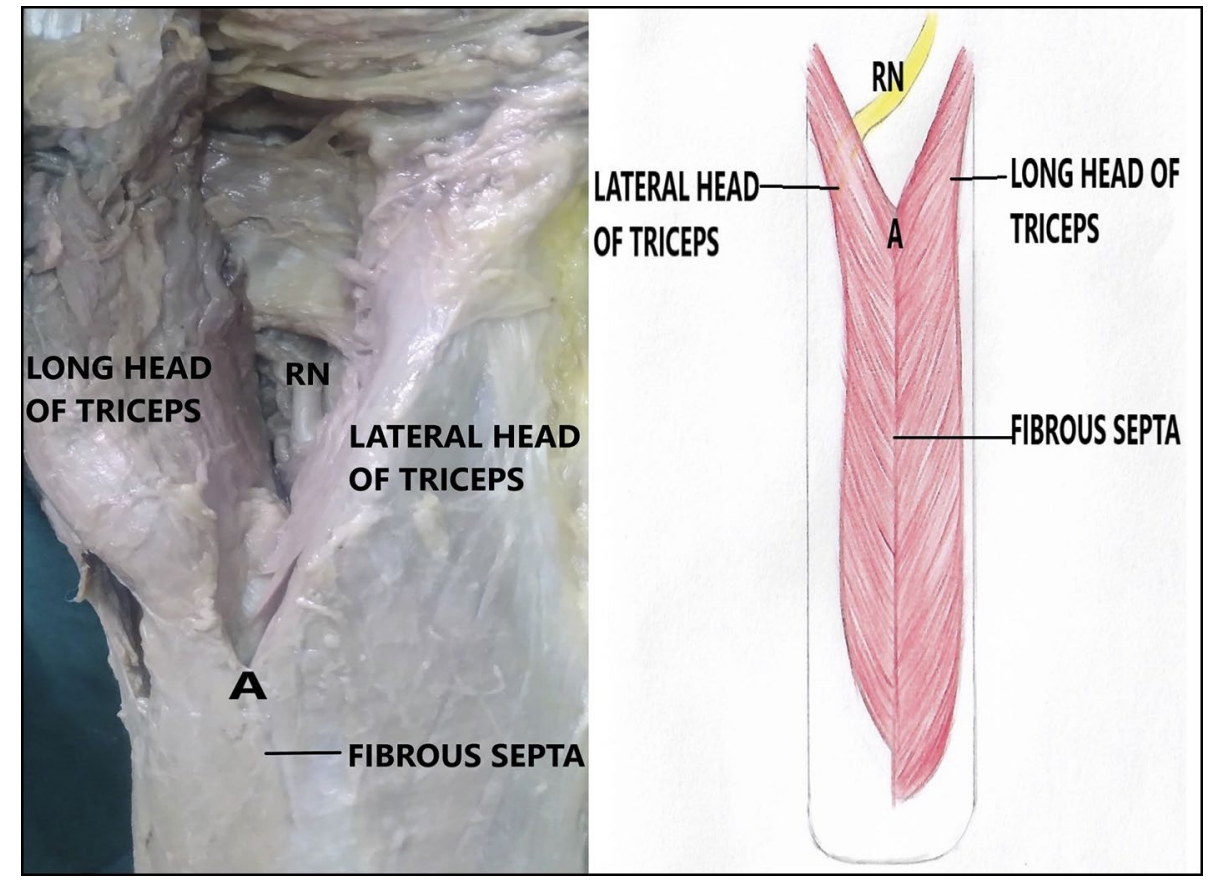

Figure 6. Tricipital aponeurosis was absent, the two heads of the triceps inserted into the fibrous intramuscular septum. No proximal apex, the meeting point of two heads of triceps with fibrous septa forms the point of confluence (A). (Schematic drawing is the mirror image of the actual photograph); $\mathrm{RN}$ - radial nerve.

of the olecranon. The percentage distribution of TA pattern did not show any gender difference (Table 1). The mean length and breadth of TA was $5.22 \pm 0.60 \mathrm{~cm}$, and $4.17 \pm 0.45 \mathrm{~cm}$, respectively. The mean distance of the RN from the point of confluence of TA (TRD, at point of confluence) and the lateral border of triceps aponeurosis (TRD, along the lateral border of TA) was $3.57 \pm 0.19 \mathrm{~cm}$ and $2.04 \pm 0.56 \mathrm{~cm}$, respectively. 
Table 1. The percentage distribution of TA shape among female and male

\begin{tabular}{lccccc}
\hline Pattern & \multicolumn{2}{c}{ Male $\mathbf{N}=\mathbf{2 3} \times \mathbf{2}=\mathbf{4 6})$} & & \multicolumn{2}{c}{ Female (N = $\mathbf{7} \times \mathbf{2}=\mathbf{1 4})$} \\
\cline { 2 - 3 } & Number & Percentage & & Number & Percentage \\
\hline I & 34 & $73.91 \%$ & & 12 & $85.71 \%$ \\
II & 9 & $19.56 \%$ & & 2 & $14.28 \%$ \\
III & 2 & $4.34 \%$ & & Nil & \\
IV & 1 & $2.17 \%$ & & Nil \\
\hline
\end{tabular}

Table 2. The morphometric measurements of tricipital aponeurosis for male and female

\begin{tabular}{|c|c|c|c|c|}
\hline \multirow[t]{3}{*}{ Morphometric variable } & \multicolumn{4}{|c|}{ Sex } \\
\hline & \multicolumn{2}{|c|}{ Male } & \multicolumn{2}{|c|}{ Female } \\
\hline & Mean & Range & Mean & Range \\
\hline TA length & 16.35 & $12.80-20.30$ & 16.96 & 15.30-20.00 \\
\hline TA breadth & 3.55 & $2.50-4.50$ & 3.60 & $2.80-4.30$ \\
\hline TRD (at point of confluence) & 3.58 & $3.00-4.00$ & 3.54 & $3.00-3.80$ \\
\hline TRD (along the lateral border of TA) & 1.98 & $1.00-3.00$ & 2.23 & $1.50-2.80$ \\
\hline
\end{tabular}

TA — tricipital aponeurosis; TRD — tricepso-radial distance

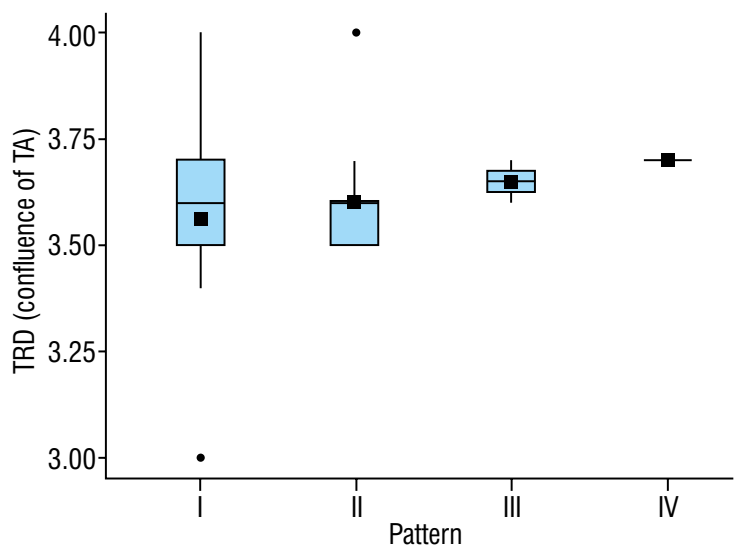

Figure 7. Boxplot depicting data set of the variables and their correlation. $X$ axis: Patterns of tricipital aponeurosis (TA); $Y$ axis: tricepso-radial distance at the point of confluence; TRD — tricepso-radial distance.

None of the measured variables showed any existing gender difference (Table 2).

Based on the morphological classification of TA, the morphometric variables (length and breadth of TA, TRDs) were examined.

The mean of TA length was $16.3 \mathrm{~cm}$ (range: 12.70$-20.30 \mathrm{~cm}$ ), $16.1 \mathrm{~cm}$ (range: $13.00-20.00 \mathrm{~cm}$ ), $17.6 \mathrm{~cm}$ (range: $16.80-18.30 \mathrm{~cm}$ ) for patterns I, II, and III, respectively.

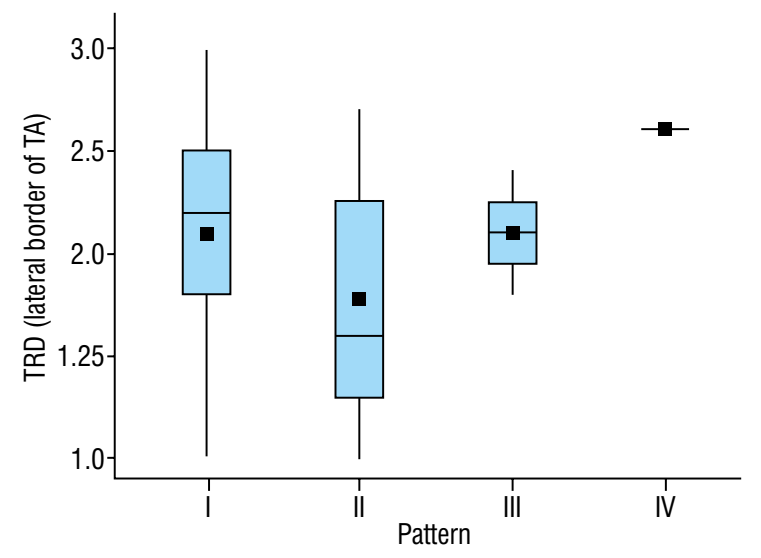

Figure 8. Boxplot depicting data set of the variables and their correlation. $X$ axis: Patterns of tricipital aponeurosis (TA); $Y$ axis: tricepso-radial distance along the lateral border of $T A$; TRD — tricepso-radıal distance.

Similarly, the mean of TA width was $4.17 \mathrm{~cm}$ (range: $2.50-4.30 \mathrm{~cm}$ ), $3.68 \mathrm{~cm}$ (range: $2.70-4.40 \mathrm{~cm}$ ), and $3.70 \mathrm{~cm}$ (range: $3.10-4.30 \mathrm{~cm}$ ) for patterns I, II, and III, respectively.

The mean distance of the RN from point of confluence (TRD at the point of confluence) was $3.56 \mathrm{~cm}$ (range: $3.00-4.00 \mathrm{~cm}$ ), $3.60 \mathrm{~cm}$ (range: $3.50-4.00 \mathrm{~cm}$ ), $3.65 \mathrm{~cm}$ (range: $3.60-3.70 \mathrm{~cm}$ ), and $3.70 \mathrm{~cm}$ for patterns I, II, III, and IV, respectively. 
Similarly, the mean distance of the RN from the lateral border of TA (TRD along the lateral border of TA) was $2.09 \mathrm{~cm}$ (range: $1.00-3.00 \mathrm{~cm}$ ), $1.77 \mathrm{~cm}$ (range: $1.00-2.70 \mathrm{~cm}$ ), $2.10 \mathrm{~cm}$ (range: $1.80-2.40 \mathrm{~cm}$ ), and $2.60 \mathrm{~cm}$ for patterns I, II, III, and IV, respectively.

A significant correlation was found between the anatomical pattern of TA and safe distance of radial nerve, i.e. TRD at point of confluence (Fig. 7) and TRD along the lateral border of TA (Fig. 8).

\section{DISCUSSION}

The radial nerve lies in close approximation with the periosteum while running through the spiral groove in the posterior compartment of the arm. Fracture of the distal humerus may either injure the RN or derange its usual course. So, identification and localisation of the $\mathrm{RN}$ is a prerequisite for surgical repair of the fractured humerus. Various authors have studied the location of $\mathrm{RN}$ in relation to the bony landmarks (lateral epicondyle, tip of acromion, tip of olecranon, and radial groove) [6, 9, 13, 14, 25, 28]. Most of these studies did not have any correlative value, with wide inter-observer variability, and are difficult for surgeons to access intraoperatively $[2,15]$. Based on cadaveric dissections, these studies show that the distance of the RN within the spiral groove from the tip of the lateral epicondyle of the humerus ranges from $10 \mathrm{~cm}$ to $18 \mathrm{~cm}$. Whereas the distance of the RN from the tip of the acromion ranges from $14 \mathrm{~cm}$ to $17 \mathrm{~cm}$. Considering the wide range of these proposed anatomic relationships, it may be cumbersome to localize the RN during the posterior approach to the humerus.

Moreover, in the setting of the fractured humerus with dislocation of the distal fragment, such anatomic relationships may not hold true [18] and it may be imprudent for a surgeon to rely on these bony landmarks to localise RN during operative fixation of fractures [1]. Therefore, in case of comminuted fracture of the humerus, a non-osseous superficial soft tissue landmark may guide the orthopaedic surgeon to identify the RN and prevent potential iatrogenic injury. Few studies are describing the relationship of the RN to superficial soft tissue landmarks like the apex of the TA, triceps tendon, the point of confluence of TA and lateral border of TA [1, 11, 13, 25]. Among these, the 'point of confluence' or 'proximal apex' of TA is an easily distinguishable and constantly available superficial soft tissue structure that may be considered as a useful landmark for surgical explora- tion of RN during posterior triceps splitting approach. In such an approach, a longitudinal incision is given along the length of the TA directed below upwards. The upward extension of the triceps split depends upon the distance between the RN and the 'point of confluence'. Various authors have measured this distance and depending on the results obtained, they have advocated a 'safe zone' for RN dissection [1, 3, $23,25]$. The apex of TA is especially helpful in localisation of radial nerve in the setting of the distal third humerus shaft fracture as this soft tissue landmark is formed by the long head and lateral head of triceps and their attachments are well proximal to the level of injury, thus, not likely to change with trauma [1]. Patra et al [22] named this distance as tricepso-radial distance (TRD at the point of confluence) and reported this as the most consistent one for RN localisation.

Few authors $[7,20,21,23]$ have reported the lateral border of the TA as a useful and reliable guide to locating the RN on the lateral aspect of the humerus in a range of positions during lateral approach. Depending upon the closest distance of the nerve from the lateral border of TA, they have also proposed a 'safe zone' for soft tissue dissection during the application of pre-contoured anatomic plates in the lateral column. So, it's a proven fact that TA is potentially a stable and reliable superficial soft tissue landmark to locate and protect the RN intraoperatively.

Reviewing literature leaves us with much uncertainty as the so-called 'safe zone' for RN varies widely among different studies. This can be due to the differences in measuring techniques, inter-observer error, sample size, ethnicity and most importantly variant patterns of the TA under study.

Variations in the morphology and morphometry of TA are not uncommon phenomenon. Most of the previous studies have focused on the relationship between RN and TA but none of them have addressed the issue of the variant anatomy of TA. In the present work, we have studied the variations in the shape of the TA and reported four variant patterns. The pattern I was reported to be the most common. Most of the previous authors, although have not spoken much about the morphological variations of TA but have described pattern I as the classical variant [24]. Any deviation in the shape of the TA, especially the shape of its 'proximal apex' or 'confluence' can literally alter the length of the triceps split and so-called 'safe zone' for RN dissection in the posterior approach. 
Study results of the current work showed variations in the mean distance of RN from point of confluence. It was highest in pattern IV, gradually decreased and became lowest in the pattern I. Although, no significant correlation was reported between TRD (at point of confluence) and TA patterns. Similarly, the mean distance between the lateral border of TA and $\mathrm{RN}$ also varies among different patterned TA but a significant correlation could not be established. The mean distance of the RN from the lateral border of TA was highest in pattern IV and lowest in pattern II. So, depending upon the values of TRD, pattern IV may be considered the safest one for RNs. In such a pattern, surgeons have more freedom of soft tissue mobilisation without injuring the nerve.

The morphometric variables of the TA (length and breadth), although varies among different patterned TA, does not seem to impact TRD or 'safe distance' directly. These values are more crucial when orthopaedic surgeons using TA as a graft for olecranon osteotomy or the TATA approach.

The intraarticular distal humerus fractures are notorious for its complication in form of stiff elbows. Such conditions can be managed through various approaches, such as Campbell's triceps aponeurosis tongue approach (TATA approach), olecranon osteotomy, and triceps reflecting anconeus pedicle approach, with each one having its share of merits and demerits [5, 27, 30]. TATA approach has shown good to excellent results. It gives stable, pain-free elbows with a good range of movement and without stiffness [12]. In this procedure, a rectangular flap of a portion of muscle along with aponeurosis is elevated from proximally to distally up to olecranon leaving a rim of aponeurosis behind for future reattachment. Underneath the triceps muscle is split and retracted medially as well as laterally. The elbow is gently flexed, while the olecranon is pulled through the aponeurotic tongue. After suturing both limbs of triceps muscles, TA was reattached to the rim of the aponeurosis. A better understanding of the length and breadth of TA is desirable for the successful placement of the implant in the TATA approach. Pattern IV although rarest, such re-constructive surgeries are not desirable in such instances.

\section{Limitations of the study}

The smaller number of female cadavers (7) than that of the males (23) did not allow us to compare our findings between sexes. Moreover, due to the non-availability of literature on this topic, we could not compare our findings with others. The interindividual variations inbuilt, body fat and body mass index can effectively influence the value of the measured variables.

\section{CONCLUSIONS}

Tricipital aponeurosis may act as a reliable and readily identifiable landmark to protect RN during surgical intervention. However, variant anatomy of TA may hinder the purpose, as the so-called 'safe zone' for radial nerve varies pattern-wise. We propose a new classification of TA anatomy. Such classification is a simpler and better way to describe TA anatomy; however, its success relies upon universal acceptance

\section{Acknowledgements}

The authors sincerely thank those who donated their bodies to science so that anatomical research could be performed. Results from such research can potentially increase mankind's overall knowledge that can then improve patient care. Therefore, these donors and their families deserve our highest gratitude

\section{Conflict of interest: None declared}

\section{REFERENCES}

1. Arora S, Goel N, Cheema GS, et al. A method to localize the radial nerve using the 'apex of triceps aponeurosis' as a landmark. Clin Orthop Relat Res. 2011; 469(9): 2638-2644, doi: 10.1007/s11999-011-1791-4, indexed in Pubmed: 21286885.

2. Artico M, Telera S, Tiengo $C$, et al. Surgical anatomy of the radial nerve at the elbow. Surg Radiol Anat. 2009; 31(2): 101-106, doi: 10.1007/s00276-008-0412-8, indexed in Pubmed: 18795220.

3. Ashfaq Hasan S, Rauls RB, Cordell CL, et al. "Zone of vulnerability" for radial nerve injury: anatomic study. J Surg Orthop Adv. 2014; 23(2): 105-110, doi: 10.3113/ jsoa.2014.0105, indexed in Pubmed: 24875341.

4. Bono C, Grossman M, Hochwald N, et al. Radial and Axillary Nerves. Clin Orthop Rel Res. 2000; 373: 259-264, doi: 10.1097/00003086-200004000-00032.

5. Bryan RS, Morrey BF. Extensive posterior exposure of the elbow. A triceps-sparing approach. Clin Orthop Relat Res. 1982(166): 188-192, indexed in Pubmed: 7083671.

6. Carlan D, Pratt J, Patterson JM, et al. The radial nerve in the brachium: an anatomic study in human cadavers. J Hand Surg Am. 2007; 32(8): 1177-1182, doi: 10.1016/j. jhsa.2006.07.001, indexed in Pubmed: 17923300.

7. Chaudhry T, Noor S, Maher B, et al. The surgical anatomy of the radial nerve and the triceps aponeurosis. Clin Anat. 2010; 23(2): 222-226, doi: 10.1002/ca.20903, indexed in Pubmed: 20014401.

8. Chou PH, Shyu JF, Ma HL, et al. Courses of the radial nerve differ between chinese and Caucasians : clinical appli- 
cations. Clin Orthop Relat Res. 2008; 466(1): 135-138, doi: 10.1007/s11999-007-0019-0, indexed in Pubmed: 18196385.

9. Clement $\mathrm{H}$, Pichler $\mathrm{W}$, Tesch NP, et al. Anatomical basis of the risk of radial nerve injury related to the technique of external fixation applied to the distal humerus. Surg Radiol Anat. 2010; 32(3): 221-224, doi: 10.1007/s00276009-0568-x, indexed in Pubmed: 19806288.

10. Cox $C L$, Riherd $D$, Tubbs RS, et al. Predicting radial nerve location using palpable landmarks. Clin Anat. 2010; 23(4): 420-426, doi: 10.1002/ca.20951, indexed in Pubmed: 20196128

11. DeFranco MJ, Lawton JN. Radial nerve injuries associated with humeral fractures. J Hand Surg Am. 2006; 31(4): 655-663, doi: 10.1016/j.jhsa.2006.02.013, indexed in Pubmed: 16632062.

12. Dev D. Results of triceps aponeurosis tongue approach for distal humerus fractures. J Med Sci Clin Res. 2019; 7(4): 134-137, doi: 10.18535/jmscr/v7i4.25.

13. Gerwin M, Hotchkiss RN, Weiland AJ. Alternative operative exposures of the posterior aspect of the humeral diaphysis with reference to the radial nerve. J Bone Joint Surg Am. 1996; 78(11): 1690-1695, doi: 10.2106/00004623199611000-00008, indexed in Pubmed: 8934483.

14. Guse TR, Ostrum RF. The surgical anatomy of the radial nerve around the humerus. Clin Orthop Relat Res. 1995(320): 149-153, indexed in Pubmed: 7586819.

15. Hackl M, Damerow $D$, Leschinger T, et al. Radial nerve location at the posterior aspect of the humerus: an anatomic study of 100 specimens. Arch Orthop Trauma Surg. 2015; 135(11): 1527-1532, doi: 10.1007/s00402-015-2300-0, indexed in Pubmed: 26254580.

16. Hoppenfeld S, deBoer P, Buckley R. Surgical Exposures in Orthopaedics: The Anatomic Approach. Lippincott, Williams \& Wilkins 2012.

17. Huttunen TT, Kannus P, Lepola V, et al. Surgical treatment of humeral-shaft fractures: a register-based study in Finland between 1987 and 2009. Injury. 2012; 43(10): 1704-1708, doi: 10.1016/j.injury.2012.06.011, indexed in Pubmed: 22771121.

18. Kamineni S, Ankem H, Patten DK. Anatomic relationship of the radial nerve to the elbow joint: clinical implications of safe pin placement. Clin Anat. 2009; 22(6): 684-688, doi: 10.1002/ca.20831, indexed in Pubmed: 19637299.

19. McCann PA, Smith GCS, Clark D, et al. The tricipital aponeurosis--a reliable soft tissue landmark for humeral plating. Hand Surg. 2015; 20(1): 53-58, doi: 10.1142/ S0218810415500070, indexed in Pubmed: 25609275.

20. McCormack RG, Brien D, Buckley RE, et al. Fixation of fractures of the shaft of the humerus by dynamic compression plate or intramedullary nail. A prospective, ran- domised trial. J Bone Joint Surg Br. 2000; 82(3): 336-339, doi: 10.1302/0301-620x.82b3.9675, indexed in Pubmed: 10813165

21. Patra A, Chaudhary P, Arora K, et al. Surgical anatomy of the radial nerve in the anterior compartment of the arm: relationship with the triceps aponeurosis. Surg Radiol Anat. 2021; 43(5): 689-694, doi: 10.1007/s00276-02102683-z, indexed in Pubmed: 33515288.

22. Patra A, Chaudhary P, Malhotra V, et al. Identification of most consistent and reliable anatomical landmark to locate and protect radial nerve during posterior approach to humerus: a cadaveric study. Anat Cell Biol. 2020; 53(2): 132-136, doi: 10.5115/acb.20.075, indexed in Pubmed: 32647080.

23. Prasad M, Issac B, Premkumar S. Anatomic landmarks to identify the radial nerve during the posterior approach of the humerus: a cadaveric study. J Clin Diag Res. 2018, doi: $10.7860 / \mathrm{jcdr} / 2018 / 37922.12275$.

24. Ring D, Gulotta L, Chin K, et al. Concomitant nonunion of the distal humerus and olecranon. J South Orthop Assoc. 2003; 12(1): 27-31, indexed in Pubmed: 12735622.

25. Seigerman DA, Choung EW, Yoon RS, et al. Identification of the radial nerve during the posterior approach to the humerus: a cadaveric study. J Orthop Trauma. 2012; 26(4): 226-228, doi: 10.1097/BOT.0b013e31821d0200, indexed in Pubmed: 21918485.

26. Shao YC, Harwood P, Grotz MRW, et al. Radial nerve palsy associated with fractures of the shaft of the humerus: a systematic review. J Bone Joint Surg Br. 2005; 87(12): 1647-1652, doi: 10.1302/0301-620X.87B12.16132, indexed in Pubmed: 16326879.

27. Tsai $\mathrm{CH}$, Fong $\mathrm{YC}$, Chen $\mathrm{YH}$, et al. The epidemiology of traumatic humeral shaft fractures in Taiwan. Int Orthop. 2009; 33(2): 463-467, doi: 10.1007/s00264-008-0537-8, indexed in Pubmed: 18414861.

28. Van Sint Jan S, Nguyen Van D, Rooze M. Quantified relationships of the radial nerve with the radial groove and selected humeral landmarks. Surg Radiol Anat. 2008; 30(8): 627-631, doi: 10.1007/s00276-008-0388-4, indexed in Pubmed: 18633562.

29. Wang JP, Shen WJ, Chen WM, et al. latrogenic radial nerve palsy after operative management of humeral shaft fractures. J Trauma. 2009; 66(3): 800-803, doi: 10.1097/ TA.0b013e31816142cf, indexed in Pubmed: 19276756.

30. Wilkinson JM, Stanley D. Posterior surgical approaches to the elbow: a comparative anatomic study. J Shoulder Elbow Surg. 2001; 10(4): 380-382, doi: 10.1067/ mse.2001.116517, indexed in Pubmed: 11517370.

31. Zlotolow DA, Catalano LW, Barron OA, et al. Surgical exposures of the humerus. J Am Acad Orthop Surg. 2006; 14(13): 754-765, doi: 10.5435/00124635-20061200000007, indexed in Pubmed: 17148623. 